\title{
Machine learning classification of conduct disorder with high versus low levels of callous-unemotional traits based on facial emotion recognition abilities
}

\author{
Ruth Pauli ${ }^{1}$ (1) - Gregor Kohls ${ }^{2,3} \cdot$ Peter Tino $^{4}$ - Jack C. Rogers ${ }^{1,5} \cdot$ Sarah Baumann ${ }^{6} \cdot$ Katharina Ackermann $^{7,8}$. \\ Anka Bernhard ${ }^{7} \cdot$ Anne Martinelli ${ }^{7} \cdot$ Lucres Jansen $^{9} \cdot$ Helena Oldenhof $^{9} \cdot$ Karen Gonzalez-Madruga $^{10}$. \\ Areti Smaragdi ${ }^{11} \cdot$ Miguel Angel Gonzalez-Torres $^{12} \cdot$ Iñaki Kerexeta-Lizeaga ${ }^{12} \cdot$ Cyril Boonmann $^{13} \cdot$ Linda Kersten $^{13}$. \\ Aitana Bigorra ${ }^{14}$. Amaia Hervas ${ }^{14}$. Christina Stadler ${ }^{13}$. Aranzazu Fernandez-Rivas ${ }^{12} \cdot$ Arne Popma $^{9}$. \\ Kerstin Konrad ${ }^{2,15} \cdot$ Beate Herpertz-DahImann $^{6} \cdot$ Graeme Fairchild $^{16} \cdot$ Christine M. Freitag $^{7} \cdot$ Pia Rotshtein $^{1}$. \\ Stephane A. De Brito ${ }^{1}$
}

Received: 25 March 2021 / Accepted: 14 July 2021 / Published online: 18 October 2021

(c) The Author(s) 2021

\begin{abstract}
Conduct disorder (CD) with high levels of callous-unemotional traits $(\mathrm{CD} / \mathrm{HCU})$ has been theoretically linked to specific difficulties with fear and sadness recognition, in contrast to $\mathrm{CD}$ with low levels of callous-unemotional traits $(\mathrm{CD} / \mathrm{LCU})$. However, experimental evidence for this distinction is mixed, and it is unclear whether these difficulties are a reliable marker of CD/HCU compared to CD/LCU. In a large sample ( $N=1263$, 9-18 years), we combined univariate analyses and machine learning classifiers to investigate whether $\mathrm{CD} / \mathrm{HCU}$ is associated with disproportionate difficulties with fear and sadness recognition over other emotions, and whether such difficulties are a reliable individual-level marker of CD/HCU. We observed similar emotion recognition abilities in $\mathrm{CD} / \mathrm{HCU}$ and $\mathrm{CD} / \mathrm{LCU}$. The $\mathrm{CD} / \mathrm{HCU}$ group underperformed relative to typically developing (TD) youths, but difficulties were not specific to fear or sadness. Classifiers did not distinguish between youths with $\mathrm{CD} / \mathrm{HCU}$ versus $\mathrm{CD} / \mathrm{LCU}$ ( $52 \%$ accuracy), although youths with $\mathrm{CD} / \mathrm{HCU}$ and $\mathrm{CD} / \mathrm{LCU}$ were reliably distinguished from TD youths (64\% and 60\%, respectively). In the subset of classifiers that performed well for youths with $\mathrm{CD} / \mathrm{HCU}$, fear and sadness were the most relevant emotions for distinguishing them from youths with CD/LCU and TD youths, respectively. We conclude that non-specific emotion recognition difficulties are common in $\mathrm{CD} / \mathrm{HCU}$, but are not reliable individual-level markers of $\mathrm{CD} / \mathrm{HCU}$ versus $\mathrm{CD} / \mathrm{LCU}$. These findings highlight that a reduced ability to recognise facial expressions of distress should not be assumed to be a core feature of $\mathrm{CD} / \mathrm{HCU}$.
\end{abstract}

Keywords Emotion recognition $\cdot$ Conduct disorder $\cdot$ Conduct problems $\cdot$ Callous-unemotional traits $\cdot$ Machine learning

\section{Introduction}

Conduct disorder (CD) is a childhood psychiatric disorder consisting of severe and persistent aggression and violations of age-appropriate social norms. A subset of youths with CD also present with high levels of callous-unemotional (CU) traits (i.e., low levels of empathy and remorse), which are the

Ruth Pauli

r.pauli@bham.ac.uk

Stephane A. De Brito s.a.debrito@bham.ac.uk

Extended author information available on the last page of the article core affective features of psychopathy [1]. This presentation is now recognised as a distinct subtype of CD [2], referred to here as $\mathrm{CD} / \mathrm{HCU}$. Youths with $\mathrm{CD} / \mathrm{HCU}$ typically exhibit more severe and instrumental aggression than youths with $\mathrm{CD}$ and lower levels of CU traits (CD/LCU) [3, 4]. According to Blair's neurocognitive model, instrumental aggression in $\mathrm{CD} / \mathrm{HCU}$ results from amygdala hypo-reactivity to facial expressions of distress, specifically fear and sadness ([5]. For typically developing (TD) youths, the distress of others is aversive and inhibits aggression, but in $\mathrm{CD} / \mathrm{HCU}$, no such deterrence occurs [5]. Blair's theory predicts that because distress cues are not as salient for youths with $\mathrm{CD} / \mathrm{HCU}$, they will have disproportionate difficulties in recognising facial expressions of distress. By contrast, there is no theoretical 
reason to hypothesise specific difficulties with distress recognition in $\mathrm{CD} / \mathrm{LCU}$.

Although emotion recognition difficulties are common in CD [6], the evidence that these difficulties are as predicted by Blair's model is mixed. Emotion recognition difficulties are not universal in CD [6,7], and while some studies implicate CU traits in this heterogeneity (e.g. [8]), others do not [9]. In this study, we first used univariate analyses to investigate whether $\mathrm{CD} / \mathrm{HCU}$ and $\mathrm{CD} / \mathrm{LCU}$ were associated with group-level differences in emotion recognition abilities, especially fear and sadness, relative to each other and to TD youths. We then used a multivariate machine learning classifier to examine whether, and to what extent, these differences provided reliable markers for distinguishing between $\mathrm{CD} /$ $\mathrm{HCU}, \mathrm{CD} / \mathrm{LCU}$, and TD at the level of individual youths.

\section{Evidence for emotion recognition difficulties in CD and its $\mathrm{CU}$ trait subtypes}

Mixed CD samples (where youths are not subtyped by CU traits) usually exhibit general difficulties in recognising emotions, which are not specific to fear and sadness, nor negative emotions more generally (e.g. [9] but [10,11]). Using a very large sample overlapping with the one used in the present study, Kohls et al. [6] reported non-specific emotion recognition difficulties in CD. However, the effect size was small, highlighting that many youths with $\mathrm{CD}$ exhibit normative emotion recognition abilities (see also [7]). These studies suggest that general difficulties should be expected in both $\mathrm{CD} / \mathrm{HCU}$ and $\mathrm{CD} / \mathrm{LCU}$, although these might be inconsistent across individuals. When $\mathrm{CD} / \mathrm{HCU}$ and $\mathrm{CD} / \mathrm{LCU}$ are considered separately, several studies report particular difficulties with negative emotions in $\mathrm{CD} / \mathrm{HCU}$ (or "affective disturbance and impulsive and conduct problems"-[12])). $\mathrm{CD} / \mathrm{HCU}$ has been associated with fear and sadness recognition difficulties over and above those seen in CD/LCU $[8,12-14]$, although one of these studies also reported difficulties with surprise [13]. Hartmann and Schwenck [15] reported that youths with $\mathrm{CD} / \mathrm{HCU}$ exhibited similar accuracy to those with $\mathrm{CD} / \mathrm{LCU}$ when identifying anger, sadness, and fear, but their reaction times were slower, suggesting a processing speed deficit (or perhaps an attentional deficit; see, e.g. [16]). Others have reported negative associations between CU or psychopathic traits and sadness, fear, and disgust recognition, as well as between antisocial behaviour and fear, anger, sadness, and disgust recognition [17, 18]. However, emotion recognition difficulties in $\mathrm{CD} / \mathrm{HCU}$ are not consistently limited to negative emotions [19], nor consistently worse than in CD/LCU [7, 11, 20]. Kohls et al. [6] also found no evidence for a correlation between CU traits and emotion recognition abilities. Indeed, a positive association between $\mathrm{CU}$ traits and fear recognition was reported in a mixed CD-and-TD sample [21]. Thus, although there are indications that $\mathrm{CD} / \mathrm{HCU}$ is linked to disproportionate difficulties in recognising negative emotions, especially fear and sadness, it is not yet clear how consistent this association is, especially across individuals.

\section{A machine learning approach}

The principal advantages of a machine learning classifier in this context are its multivariate nature, combined with its ability to make predictions about individuals. By projecting data into multi-dimensional feature space (with each predictor variable, or feature, constituting a different dimension), a classifier is able to construct a decision boundary that optimally separates individuals of different classes within this space. In doing so, it naturally takes into account all features and their interactions simultaneously. This decision boundary is then used to predict the class membership (e.g. CD subtype) of previously unseen individuals, based on their location in the feature space. Furthermore, in-built feature selection or ranking procedures can provide additional information about the relative importance of each feature for classification, within the multivariate context. A machine learning approach therefore complements a standard univariate analysis, which is hypothesis-driven and uses an explicit statistical model to address a specific hypothesis. In this case, we use a univariate approach to determine whether or not there are group-level differences in emotion recognition abilities in $\mathrm{CD} / \mathrm{HCU}$ and $\mathrm{CD} / \mathrm{LCU}$, especially for fear and sadness, and a machine learning classifier to quantify how reliable any such differences are as markers of CD subtype in individual youths.

The classifier used here, Angle-based Generalised Matrix Learning Vector Quantisation (Angle-GMLVQ; [22]), has the advantage of an in-built feature relevance procedure, which can be used to rank features for their relevance to the classification decision. In addition, Angle-GMLVQ is sensitive to the relative differences (angles) between features rather than their absolute magnitude. This makes it especially sensitive to different patterns of performance across emotions (e.g. specific difficulties with fear and sadness) rather than general emotion recognition difficulties. For an example of this approach, please see [23], where we used Angle-GMLVQ to classify CD/HCU, CD/LCU, and TD based on differences in parenting behaviours.

\section{Hypotheses}

We used a large, well-characterised sample of youths with CD (FemNAT-CD) [24] to compare facial emotion recognition abilities in youths with $\mathrm{CD} / \mathrm{HCU}$, youths with $\mathrm{CD} / \mathrm{LCU}$, and TD youths. First, we hypothesised that general emotion recognition performance (i.e. across all emotions) would be poorest in youths with $\mathrm{CD} / \mathrm{HCU}$, followed by youths with 
$\mathrm{CD} / \mathrm{LCU}$ and then TD youths. Second, we tested the hypothesis that youths with $\mathrm{CD} / \mathrm{HCU}$ have specific difficulties with fear and sadness recognition, over and above any general difficulties. We also included sex as a factor in these analyses, to check whether any group differences were consistent across the sexes. These two hypotheses were investigated using univariate analyses (ANCOVA).

Next, we used Angle-GMLVQ to quantify the reliability of emotion recognition differences as markers of CD subtype. Although machine learning classification is not fundamentally a hypothesis-driven approach, we made several predictions about classifier performance based on our earlier hypotheses. First, based on group differences in performance, we predicted that classifier performance would exceed chance level (50\% accuracy) when distinguishing between any two classes (e.g. $\mathrm{CD} / \mathrm{HCU}$ from $\mathrm{CD} / \mathrm{LCU}, \mathrm{CD} /$ HCU from TD, etc.), and would be highest when distinguishing $\mathrm{CD} / \mathrm{HCU}$ from TD. In line with our second hypothesis regarding $\mathrm{CD} / \mathrm{HCU}$ and fear and sadness, we predicted that recognition of fear and sadness might be particularly relevant as markers of $\mathrm{CD} / \mathrm{HCU}$. Related to this prediction, we investigated whether a $\mathrm{CD} / \mathrm{HCU}$-against-TD and a $\mathrm{CD} /$ LCU-against-TD classifier would outperform a CD-againstTD classifier (where the $\mathrm{CD}$ group consisted of all youths with $\mathrm{CD}$ ). If $\mathrm{CD}$ subtypes are indeed characterised by different patterns of difficulties, then distinguishing them from TD should be easier when the distinction between subtypes (i.e. $\mathrm{CD} / \mathrm{HCU}$ vs. $\mathrm{CD} / \mathrm{LCU}$ ) is made.

\section{Methods}

\section{Participants}

Participants were selected from the FemNAT-CD dataset. Ethical approval details are provided in supplementary materials. Exclusion criteria were an IQ below 70, a history of manic or psychotic episodes, autism, or neurological or genetic disorders. In addition to these criteria, TD youths were also required to have no history of externalising disorders and no current psychiatric disorders.

Data on emotion recognition ability and CU traits were available for 1462 youths. Consistent with our recent work on this dataset [23], youths with CD were included in the $\mathrm{CD} / \mathrm{HCU}$ group if their total score on the Inventory of Callous-Unemotional traits (ICU; [25]) was in the first tertile for youths with $\mathrm{CD}$ (a score of 39 or above), and in the $\mathrm{CD} / \mathrm{LCU}$ group if their score was in the third tertile (29 or below). One hundred and ninety-nine participants with $\mathrm{CD}$ and second-tertile $\mathrm{CU}$ scores were excluded because they did not meet the criteria for either the $\mathrm{CD} / \mathrm{HCU}$ or $\mathrm{CD} / \mathrm{LCU}$ group. A small number of TD participants also had CU scores in the first tertile $(n=13)$ or second tertile $(n=44)$, but were included in the sample because they were typically developing according to the FemNAT-CD criteria, and excluding these participants did not change the pattern of results (analyses available from the authors on request). The final sample therefore consisted of 1263 participants: 248 with CD/HCU (153 females), 230 with CD/LCU (130 females), and 785 TD youths (523 females). ${ }^{1}$ For one of the Angle-GMLVQ classification analyses, the CD/HCU and $\mathrm{CD} / \mathrm{LCU}$ groups were combined into a single $\mathrm{CD}$ group ( $n=478$ ), which we refer to as the 'CD group'. Demographic differences between excluded and included participants are provided in supplementary materials.

\section{Questionnaire and interview measures}

$\mathrm{CD}$ and other relevant disorders were assessed by trained researchers, via separate clinical interviews with participants and their parents or carers, using the Schedule for Affective Disorders and Schizophrenia in School-Age Children: Present and Lifetime Version (K-SADS-PL) [26]. Number of CD symptoms reflects the most serious lifetime episode. Where discrepancies arose between parent and youth ratings, interviewers discussed all available evidence to come to a best estimate. Other disorders assessed with the K-SADSPL were attention-deficit/hyperactivity disorder, anxiety, depression, and substance use disorder. These disorders were controlled for in an additional set of analyses (see supplementary materials). To provide a more comprehensive clinical picture, we also report K-SADS-PL oppositional defiant disorder symptoms, although these did not form part of our analyses. CU traits were assessed with the ICU ([25]. This is a 24-item parent-report measure consisting of three subscales (callous, uncaring, and unemotional) plus a total score, which we used in the current study. Reliability for the ICU in the current sample was good (Cronbach's a for total score $=0.93$ ). Verbal, performance, and total IQ were estimated with the Wechsler Intelligence Scales [27, 28]. For completeness, we also report pubertal development stage as assessed with the Pubertal Development Scale (PDS) [29]. Socioeconomic status (SES) was assessed with a measure based on parental income, education level, and occupation, normalised for the country in which the participant lived (see [23]). Imputation of missing data is described in supplementary materials.

\footnotetext{
${ }^{1}$ Of the 1263 participants in the current study, 1081 participants (372 with CD) were included in the studies by Kohls et al. [6], [7]
} and 182 (106 with CD) were not. 


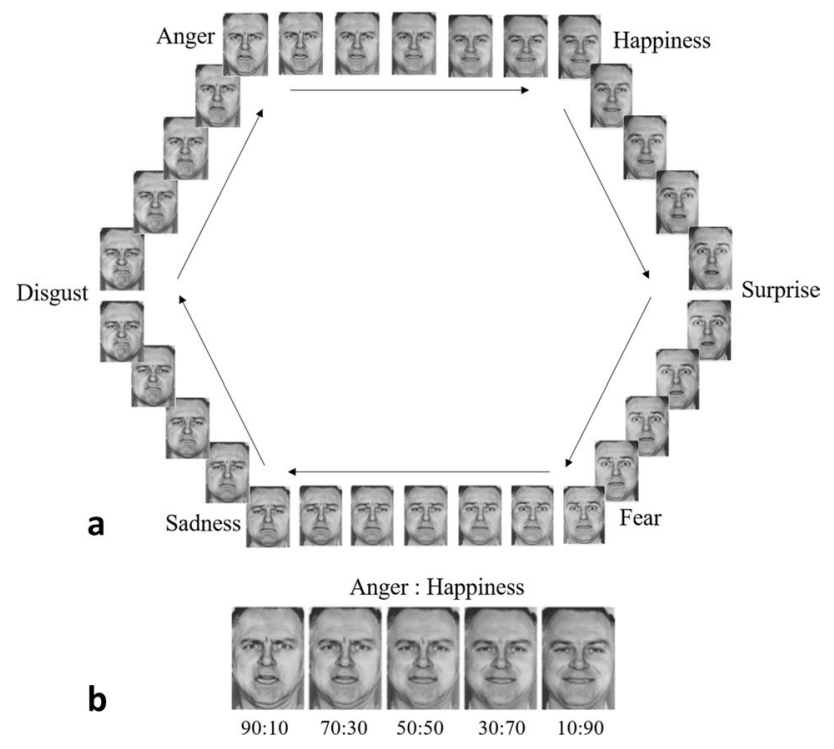

Fig. 1 Stimuli used in the Emotion Hexagon Task. a The complete set of blended expressions arranged in a hexagon. The six basic emotions lie on the vertices adjacent to their most easily confused emotion (except anger-happiness, which adjoin to complete the hexagon). b An example of the blended expressions for the anger-to-happiness continuum, with ratios labelled

\section{Emotion hexagon task}

Emotion recognition ability was assessed with the Emotion Hexagon task [30]. Full details are given in supplementary materials. Briefly, participants were presented with blended expressions of two emotions, in the ratios of 90:10, 70:30, and 50:50 (see Fig. 1). Expressions were presented individually, and participants selected with a mouse click one of six labels (see Fig. 1a) that best described the expression. The 'dominant' emotion was considered the correct response. For example, 'anger' was the correct response to an expression that was a 90:10 or 70:30 blend of anger:happiness, while 'happiness' was the correct response to an expression that was a 30:70 or 10:90 blend of anger:happiness. 50:50 blends were not scored, as there was no 'correct' answer for these expressions. For each participant, we calculated the percentage recognition accuracy for each emotion at its highest intensity (i.e. its 90:10 ratio, or $90 \%$, presentations) and its lowest (70:30, or 70\%) intensity. We refer to these as the 'high intensity' and 'low intensity' of each emotion. We considered anger, disgust, fear, and sadness to be negative emotions [31], but only fear and sadness to be 'distress' emotions, in line with their theoretical role in $\mathrm{CD} / \mathrm{HCU}$ [5].

\section{Univariate analysis}

Interactions between group, emotion, intensity (high/ low), and sex were investigated using a repeated measures
ANCOVA (using IBM SPSS Statistics 25), with group and sex as between-subject factors and emotion and intensity as within-subject factors. Site of data collection was included as a factor of no interest, and grand-mean-centred age, total IQ, and SES were entered as covariates. Analysis of sex differences was exploratory, motivated by previous mixed evidence as to whether females with $\mathrm{CD}$ exhibit the same difficulties as males $[6,10]$.

\section{Classification analysis}

The classification analyses used features derived from the 12 measures of Emotion Hexagon task performance (i.e. accuracy for six emotions at two intensity levels) to predict group status (i.e. classes were CD/HCU, CD/LCU, TD etc.). Since covariates cannot be controlled for in classification analyses, we regressed out variance associated with age, IQ, site of data collection, sex, and SES from each of the 12 measures of task performance. The final features were, thus, the standardised residual mean accuracies for each emotion at its high and low intensity levels. To assess the performance for each pair of groups of interest separately, four Angle-GMLVQ models were created with two classes each:

1. 1.CD against TD ('CD-TD' model)

2. 2.CD/HCU against TD ('HCU-TD' model)

3. 3.CD/LCU against TD ('LCU-TD' model)

4. 4.CD/HCU against CD/LCU ('HCU-LCU' model)

For each model, 100 separate classifiers were trained and tested using data re-sampling (see supplementary materials for a full description of the training and testing procedure). After training and testing each of the four models 100 times, mean performance measures were calculated for each model. Performance of the CD-TD, HCU-TD, and LCU-TD models were then compared using an ANOVA (the HCU-LCU model was not included in this ANOVA, because comparisons with the HCU-LCU model were not relevant to hypotheses). Model performance was assessed using macro-averaged accuracy (adapted from [32, 33]). This is the mean of the accuracies for each class, and is not skewed by superior performance for the larger class when class sizes are imbalanced. Positive predictive value (PPV), negative predictive value (NPV), true-positive rate (TPR), and true-negative rate (TNR) are also reported. These represent the proportion of positive classifications (e.g. $\mathrm{CD} / \mathrm{HCU}$ ) that are true positives (PPV), the proportion of negative classifications (e.g. TD) that are true negatives (NPV), the proportion of positives (i.e. cases) that are correctly classified as positives (TPR), and the proportion of negatives that are correctly classified as negatives (TNR). 
Table 1 Demographic and clinical characteristics (mean (95\% confidence intervals of the mean) unless stated otherwise)

\begin{tabular}{|c|c|c|c|c|}
\hline Measures & $\mathrm{CD} / \mathrm{HCU}(n=248)$ & $\mathrm{CD} / \mathrm{LCU}(n=230)$ & $\mathrm{TD}(n=785)$ & Test statistic (p), effect size \\
\hline Age (years) & $14.14^{\mathrm{a}}(13.86,14.41)$ & $14.30^{\mathrm{a}}(13.98,14.62)$ & $13.95^{\mathrm{a}}(13.78,14.13)$ & $F=1.94(0.14), \eta_{\mathrm{p}}^{2}=0.003$ \\
\hline Females $(\%)$ & $61.69^{\mathrm{a}, \mathrm{b}}$ & $56.52^{\mathrm{a}}$ & $66.62^{\mathrm{b}}$ & $\chi^{2}=8.46(0.02), \Phi_{c}=0.08$ \\
\hline SES & $-0.38^{\mathrm{a}}(-0.51,-0.26)$ & $-0.29^{\mathrm{a}}(-0.41,-0.16)$ & $0.33^{\mathrm{b}}(0.26,0.39)$ & $F=70.29(<0.001), \eta_{\mathrm{p}}^{2}=0.11$ \\
\hline PDS pubertal stage & $3.60^{\mathrm{a}}(3.47,3.72)$ & $3.55^{\mathrm{a}}(3.41,3.7)$ & $3.52^{\mathrm{a}}(3.44,3.59)$ & $F=0.53(0.59), \eta_{\mathrm{p}}^{2}=0.001$ \\
\hline Performance IQ & $97.21^{\mathrm{a}}(95.31,99.11)$ & $96.74^{\mathrm{a}}(94.77,98.7)$ & $103.37^{\mathrm{b}}(102.29,104.45)$ & $F=25.56(<0.001), \eta_{\mathrm{p}}^{2}=.04$ \\
\hline Verbal IQ & $92.83^{\mathrm{a}}(91.1,94.57)$ & $92.89^{\mathrm{a}}(90.9,94.87)$ & $103.52^{\mathrm{b}}(102.37,104.66)$ & $F=67.27(<0.001), \eta_{\mathrm{p}}^{2}=0.10$ \\
\hline Total IQ & $95.26^{\mathrm{a}}(93.76,96.76)$ & $94.95^{\mathrm{a}}(93.26,96.63)$ & $103.73^{\mathrm{b}}(102.79,104.67)$ & $F=63.93(<0.001), \eta_{\mathrm{p}}^{2}=0.09$ \\
\hline ICU callous & $18.15^{\mathrm{a}}(17.54,18.75)$ & $6.40^{\mathrm{b}}(5.96,6.84)$ & $4.28^{\mathrm{c}}(4.05,4.5)$ & $F=1417.01(<0.001), \eta_{\mathrm{p}}^{2}=.70$ \\
\hline ICU uncaring & $18.33^{\mathrm{a}}(17.98,18.69)$ & $10.20^{\mathrm{b}}(9.69,10.71)$ & $7.74^{\mathrm{c}}(7.45,8.03)$ & $F=703.87(<0.001), \eta_{\mathrm{p}}^{2}=0.53$ \\
\hline ICU unemotional & $9.73^{\mathrm{a}}(9.36,10.09)$ & $5.11^{\mathrm{a}}(4.76,5.46)$ & $4.83^{\mathrm{b}}(4.64,5.03)$ & $F=297.88(<0.001), \eta_{\mathrm{p}}^{2}=0.32$ \\
\hline ICU total & $46.21^{\mathrm{a}}(45.4,47.01)$ & $21.71^{\mathrm{b}}(20.96,22.46)$ & $16.84^{\mathrm{c}}(16.29,17.39)$ & $F=1553.34(<0.001), \eta_{\mathrm{p}}^{2}=0.71$ \\
\hline CD symptoms* & $5.78^{\mathrm{a}}(5.48,6.08)$ & $5.03^{\mathrm{b}}(4.69,5.38)$ & $0.09^{\mathrm{c}}(0.06,0.11)$ & $F=1753.42(<0.001), \eta_{\mathrm{p}}^{2}=0.74$ \\
\hline ODD symptoms* & $6.41^{\mathrm{a}}(6.17,6.64)$ & $5.31^{\mathrm{b}}(5.02,5.6)$ & $0.08^{\mathrm{c}}(0.06,0.11)$ & $F=2926.52(<0.001), \eta_{p}^{2}=0.82$ \\
\hline ADHD symptoms* & $7.83^{\mathrm{a}}(6.97,8.69)$ & $6.03^{\mathrm{b}}(5.21,6.85)$ & $0.11^{\mathrm{c}}(0.07,0.15)$ & $F=428.60(<0.001), \eta_{\mathrm{p}}^{2}=0.41$ \\
\hline GAD diagnosis $(\%)^{*}$ & $17.74^{\mathrm{a}}$ & $15.65^{\mathrm{a}}$ & $1.91^{\mathrm{b}}$ & $\chi^{2}=94.63(<0.001), \Phi_{c}=0.27$ \\
\hline MDD diagnosis $(\%)^{*}$ & $25.00^{\mathrm{a}}$ & $23.91^{\mathrm{b}}$ & $1.66^{\mathrm{b}}$ & $\chi^{2}=167.72(<0.001), \Phi_{c}=0.36$ \\
\hline SUD diagnosis $(\%)^{*}$ & $21.77^{\mathrm{a}}$ & $17.39^{\mathrm{a}}$ & $0.13^{b}$ & $\chi^{2}=166.33(<0.001), \Phi_{c}=0.36$ \\
\hline
\end{tabular}

$C D / H C U$ conduct disorder with high levels of callous-unemotional traits. $C D / L C U$ conduct disorder with low levels of callous-unemotional traits. $T D$ typically developing. PDS Pubertal Development Scale (self-report). ICU, Inventory of Callous-Unemotional Traits. SES socioeconomic status. $C D$ conduct disorder. $O D D$ oppositional defiant disorder. $A D H D$ attention-deficit/hyperactivity disorder. $G A D$ generalised anxiety disorder. $M D D$ major depressive disorder. $S U D$ substance use disorder. $\eta 2 p$, partial eta squared. $\Phi c$, Cramer's phi. Groups with different superscript indices differ significantly in post hoc comparisons $(p<0.05$, Bonferroni corrected)

*Assessed using K-SADS, Schedule for Affective Disorders and Schizophrenia for School-age Children: Present and Lifetime Version (lifetime maximum symptoms/diagnosis)

\section{Feature relevance}

The Angle-GMLVQ classifier generates a feature relevance score for each feature, for each trained classifier. The relevance score of a feature is a non-negative number that quantifies the importance of that feature for the given classification task. The relevance scores are normalised to sum to 1 across all features. In this way, the relevance scores learned in the 100 classifiers are directly comparable. The procedure described above thus generates 100 feature relevance scores for each feature, for each model. However, some of these classifiers will fail to distinguish between the groups, and the feature relevance scores from these models are, thus, not informative. We therefore discarded relevance scores from classifiers that did not achieve at least 50\% macro-averaged accuracy. For each model, mean relevance scores across the retained classifier models were then calculated for each feature, and compared with a one-way ANOVA. We note that such feature score learning is effectively performing feature selection [34].

\section{Supplementary analyses}

We conducted additional analyses to examine the correlations between $\mathrm{CD}$ symptoms, $\mathrm{CU}$ traits, and emotion recognition ability within each group. We also repeated the ANCOVAs and machine learning analyses after controlling for comorbid diagnoses (attention-deficit/hyperactivity disorder, depression, anxiety, and substance use disorder; note that youths with autism were excluded from this study). Finally, we investigated group differences in the number of remote prototype errors, that is, mislabelling an emotion as a non-adjacent emotion rather than an emotion with which the target was blended. Results of these analyses are reported in supplementary materials.

Finally, we also present results for two additional sets of classification analyses. First, we repeated the classification analyses described above with a support vector machine (SVM), to check that performances were comparable with this more established classifier. Second, we repeated the Angle-GMLVQ analyses using 23 instead of 12 features. These 23 features captured the percentage of trials in which each emotion at each intensity level was mislabelled as each of the other emotions (e.g. low-intensity anger mislabelled as happiness, low-intensity anger mislabelled as sadness, etc.). 
a

Performance by Group
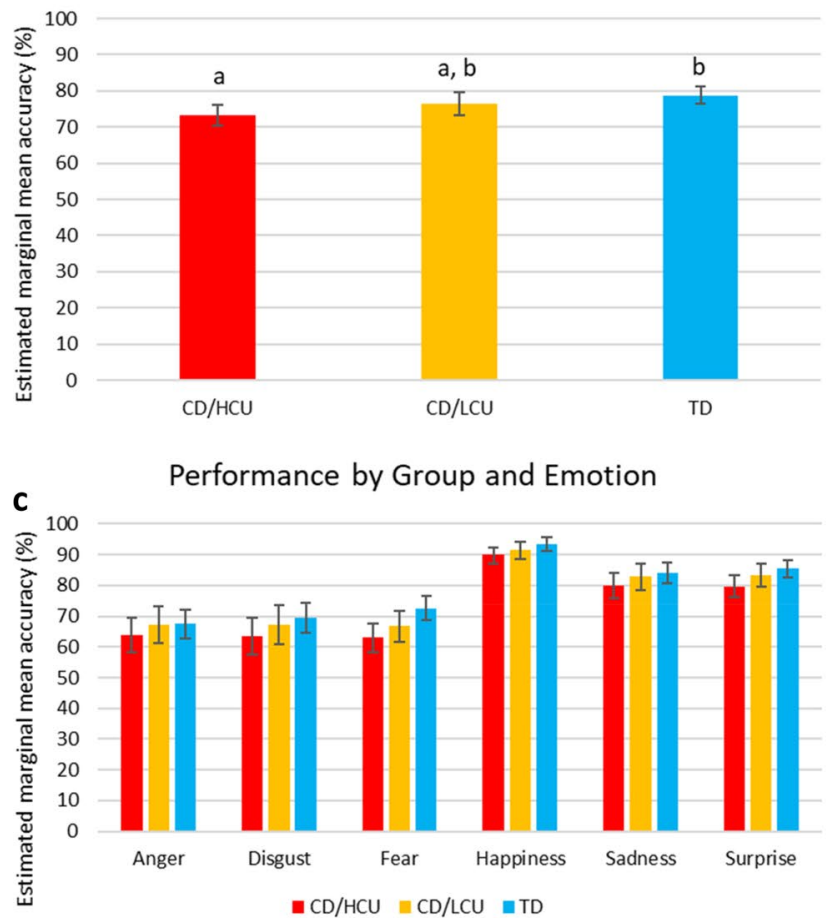

Fig. 2 Emotion Hexagon recognition task performance by (a) emotion, (b) group, (c) group and sex, and (d) group and emotion. Different letters $(\mathbf{a}, \mathbf{b}, \mathbf{c})$ above columns indicate significant differences in post hoc pairwise comparisons $(p<0.05$, Bonferroni corrected).

\section{Results}

\section{Demographic and clinical characteristics}

Group differences in demographic and clinical characteristics are displayed in Table 1. All three groups differed in CD symptoms and $\mathrm{CU}$ trait severity, with the $\mathrm{CD} / \mathrm{HCU}$ group exhibiting the most severe presentation, as expected. There were also group differences in the number of participants per group recruited from each site $\left(\chi^{2}=48.00, p<0.001\right.$, $\Phi_{\mathrm{c}}=0.14$; see supplementary materials).

\section{Group differences in emotion recognition}

The ANCOVA revealed a significant main effect of group $\left(F_{(2,1130)}=11.12, p<0.001, \eta_{p}^{2}=0.02\right)$, as illustrated in Fig. 2a. The $\mathrm{CD} / \mathrm{HCU}$ group performed significantly worse than the TD group, but the CD/LCU group did not differ from either group. Given this overlap, we repeated the ANCOVA with both CD subtypes combined into one group (i.e. $\mathrm{CD}$ versus $\mathrm{TD}$ ) to check whether $\mathrm{CD}$ as a whole was associated with poorer emotion recognition. The second ANCOVA confirmed that youths with CD exhibited poorer b Performance by Emotion
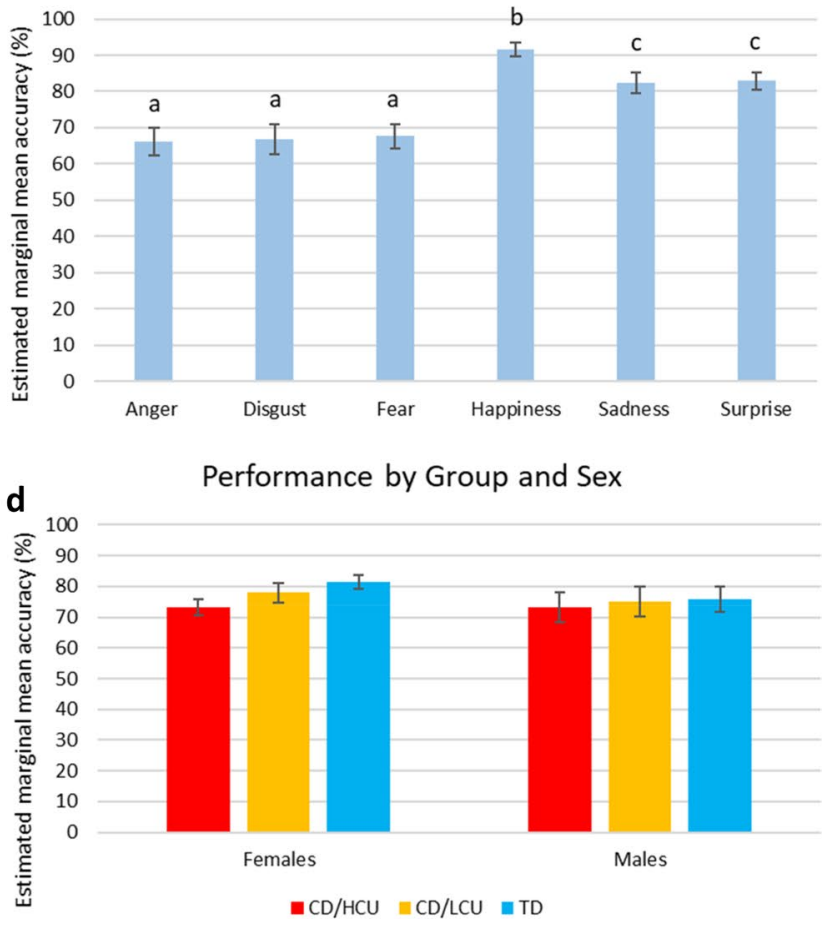

Pairwise comparisons were not conducted for panels c) and d) due to the absence of significant interactions. Error bars represent 95\% confidence intervals of the mean

performance than TD youths $\left(\mathrm{F}_{(1,1141)}=21.62, p<0.001\right.$, $\eta_{\mathrm{p}}^{2}=0.02$ ).

There was also a significant main effect of emotion $\left(\mathrm{F}_{(3.71,4190.00)}=55.5, p<0.001, \eta_{\mathrm{p}}^{2}=0.05\right)$, as illustrated in Fig. 2b. Across the full sample, recognition accuracy was highest for happiness, followed by sadness and surprise, and then anger, disgust, and fear. However, the interaction between group and emotion was not significant $\left(\mathrm{F}_{(7.42,4190.99)}=0.62, p=0.75, \eta_{\mathrm{p}}^{2}=0.001\right)$, suggesting that the underperformance of the $\mathrm{CD} / \mathrm{HCU}$ group was not disproportionate for fear and sadness (or indeed negative emotions more generally; Fig. 2c).

We did not formulate a priori hypotheses regarding emotion intensity, but noted that recognition accuracy was better for high-intensity than for low-intensity emotions $\left(\mathrm{F}_{(1,1130)}=80.51, p<0.001, \eta_{\mathrm{p}}^{2}=0.07\right)$. Interestingly, the performance gap between the $\mathrm{CD} / \mathrm{HCU}, \mathrm{CD} / \mathrm{LCU}$, and TD groups widened slightly for high-intensity emotions (group by intensity interaction: $\mathrm{F}_{(2,1130)}=3.66, p=0.03, \eta_{\mathrm{p}}^{2}=0.01$ ), suggesting that the TD group, and to a lesser extent also the $\mathrm{CD} / \mathrm{LCU}$ group, benefited more from the increased expression intensity than the $\mathrm{CD} / \mathrm{HCU}$ group. However, the threeway interaction between group, emotion, and intensity was not significant $\left(\mathrm{F}_{(9.24,5218.77)}=1.33, p=0.21, \eta_{\mathrm{p}}^{2}=0.002\right)$, 
Table 2 Angle-GMLVQ model performance (mean (95\% confidence intervals of the mean))

\begin{tabular}{lllcrc}
\hline & CD-TD & HCU-TD & LCU-TD & F $(\mathrm{p}), \eta_{\mathrm{p}}^{2}$ & HCU-LCU \\
\hline $\begin{array}{l}\text { Accuracy (macro- } \\
\text { averaged) }\end{array}$ & $0.62^{\mathrm{a}}(0.62,0.63)$ & $0.64^{\mathrm{b}}(0.64,0.65)$ & $0.60^{\mathrm{c}}(0.60,0.61)$ & $33.69(<0.001), .19$ & $0.52(0.51,0.53)$ \\
PPV & $0.54^{\mathrm{a}}(0.53,0.55)$ & $0.40^{\mathrm{b}}(0.39,0.41)$ & $0.33^{\mathrm{c}}(0.33,0.34)$ & $718.60(<0.001), .83$ & $0.53(0.52,0.54)$ \\
NPV & $0.71^{\mathrm{a}}(0.71,0.72)$ & $0.84^{\mathrm{b}}(0.83,0.84)$ & $0.83^{\mathrm{c}}(0.83,0.83)$ & $1123.18(<0.001), .88$ & $0.50(0.49,0.51)$ \\
TPR & $0.51^{\mathrm{a}}(0.50,0.52)$ & $0.54^{\mathrm{b}}(0.52,0.55)$ & $0.51^{\mathrm{a}}(0.49,0.52)$ & $6.64(0.002), 0.04$ & $0.45(0.43,0.46)$ \\
TNR & $0.74^{\mathrm{a}}(0.73,0.74)$ & $0.75^{\mathrm{a}}(0.74,0.75)$ & $0.70^{\mathrm{b}}(0.69,0.71)$ & $34.14(<0.001), 0.19$ & $0.59(0.57,0.60)$ \\
\hline
\end{tabular}

$C D-T D$ conduct disorder-typically developing model. $H C U-T D$ high callous-unemotional-typically developing model. $L C U-T D$ low callousunemotional-typically developing model. $P P V$ positive predictive value. $N P V$ negative predictive value. $T P R$ true-positive rate. $T N R$ true-negative rate. $\eta 2 p$ partial eta squared. Groups with different superscript indices differ significantly in post hoc comparisons $(p<0.05$, Bonferroni corrected). Note that the HCU-LCU model (column 6) was not included in statistical tests as comparisons between this and other models were not relevant to hypotheses

confirming that this pattern did not reflect specific difficulties with negative emotions at either intensity in the $\mathrm{CD} /$ HCU group.

There were small but significant correlations between age and sex (Pearson correlation: $r=-0.09, p=0.003$ ) and between sex and total IQ $(r=0.07, p=0.02)$, with boys tending to be younger than girls and to have higher IQs. Consequently, sex effects must be interpreted with extreme caution. However, investigation of sex differences revealed that female youths significantly outperformed male youths, as evidenced by a main effect of $\operatorname{sex}\left(\mathrm{F}_{(1,1130)}=5.88, p=0.02\right.$, $\left.\eta_{\mathrm{p}}^{2}=0.005\right)$. Although differences between the $\mathrm{CD} / \mathrm{HCU}$, $\mathrm{CD} / \mathrm{LCU}$, and TD groups were slightly larger for females than males (Fig. 2d), this group by sex interaction was not significant $\left(\mathrm{F}_{(2,1130)}=0.44, p=0.65, \eta_{\mathrm{p}}^{2}=0.001\right)$, confirming that the pattern of group differences was similar for both sexes. In summary, in these univariate analyses, group differences in emotion recognition abilities were not driven by difficulties with specific emotions such as fear and sadness.

\section{Classifier performance}

We first compared performance for the CD-TD, HCU-TD, and LCU-TD models (Table 2). Only the HCU-TD model achieved higher accuracy than the CD-TD model, with the LCU-TD model achieving the lowest accuracy of these three models. Nonetheless, all performed significantly above chance (i.e. $50 \%$; binomial tests: $p<0.001, p<0.001$, and $p=0.002$, respectively). Notably, TD youths were consistently more likely to be classified correctly (TNRs) than youths with $\mathrm{CD} / \mathrm{HCU}$ or $\mathrm{CD} / \mathrm{LCU}$ (TPRs), suggesting 'normative' performance in approximately $50 \%$ of youths with $\mathrm{CD}$ regardless of $\mathrm{CU}$ traits. Finally, the performance of the HCU-LCU model was at chance level (52\%; binomial test, $p=0.42$ ), indicating that emotion recognition difficulties were not a reliable marker of subtype within $\mathrm{CD}$. The pattern of results was similar for the additional sets of classification analyses, using an enlarged set of 23 features for the a

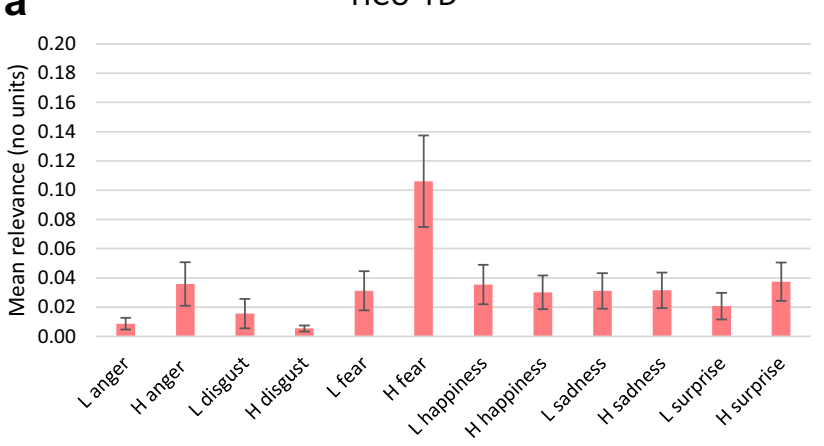

b

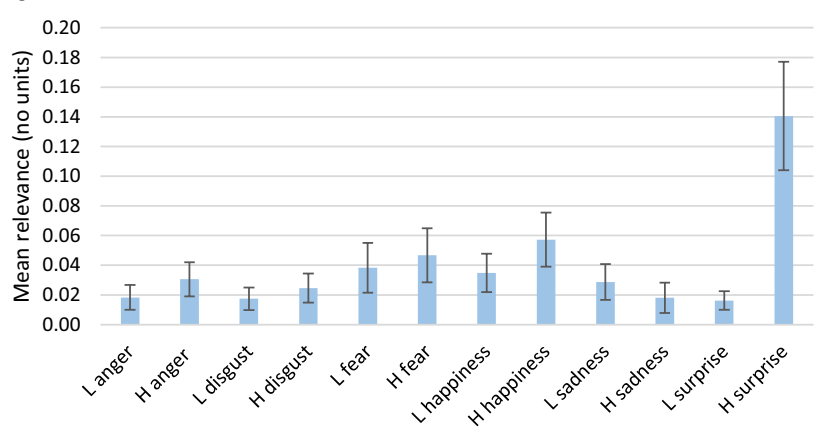

C

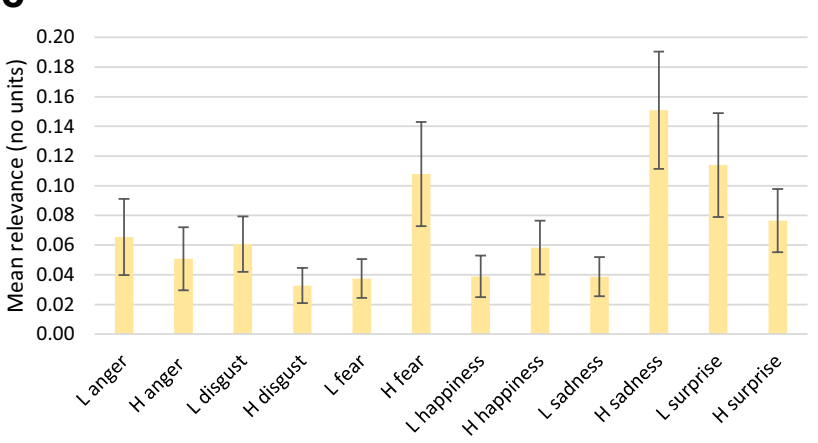

Fig. 3 Mean feature relevance scores for (a) the HCU-TD model, (b) LCU-TD model, and (c) HCU-LCU model. Error bars represent 95\% confidence intervals of the mean. $L=$ low intensity, $H=$ high intensity 
Angle-GMLVQ classifier and repeating the main analyses with SVM classifiers (see supplementary materials).

\section{Feature relevance}

Mean feature relevance scores for the HCU-TD, LCU-TD, and HCU-LCU models are displayed in Fig. 3. Feature relevance scores were not normally distributed and were thus compared with Kruskal-Wallis (non-parametric) ANOVAs. For the HCU-TD model, the most relevant feature was high-intensity fear, which differed significantly from all other emotions $\left(\mathrm{H}_{(11)}=139.2, p<0.001\right.$ : post hoc Bonferroni-corrected comparison of mean ranks, all $p<0.05$ ). For the LCU-TD model, the most relevant feature was high-intensity surprise, which was significantly more relevant than all other features except high-intensity happiness $\left(\mathrm{H}_{(11)}=101.49, p<0.001\right.$ : post hoc Bonferroni-corrected comparison of mean ranks, all $p<0.05$ except comparison with high-intensity happiness). For the HCU-LCU model, the most relevant feature was high-intensity sadness, which was significantly more relevant than anger (both intensities), high-intensity disgust, and low-intensity fear, happiness, and sadness $\left(\mathrm{H}_{(11)}=51.93, p<0.001\right.$ : post hoc Bonferroni-corrected comparison of mean ranks, $p<0.05)$.

\section{Discussion}

The aim of this study was to investigate whether $\mathrm{CD} / \mathrm{HCU}$ and $\mathrm{CD} / \mathrm{LCU}$ are associated with differences in emotion recognition abilities, especially for fear and sadness, and whether any such difficulties are sufficiently reliable to act as markers for $\mathrm{CD}$ subtype in individual youths. First, we hypothesised that overall emotion recognition ability would be lowest in youths with $\mathrm{CD} / \mathrm{HCU}$, with youths with $\mathrm{CD} /$ LCU performing at an intermediate level between youths with $\mathrm{CD} / \mathrm{HCU}$ and TD youths. Evidence supporting this hypothesis was somewhat weak. The direction of group differences was as predicted, but only the $\mathrm{CD} / \mathrm{HCU}$ and TD groups differed significantly in univariate analyses. Despite this, and although classifier performance was not strong, the Angle-GMLVQ classifier was able to discriminate both youths with $\mathrm{CD} / \mathrm{HCU}$ and youths with $\mathrm{CD} / \mathrm{LCU}$ from TD youths at above-chance accuracy levels. Second, we hypothesised that $\mathrm{CD} / \mathrm{HCU}$ would be associated with particular difficulties with fear and sadness. Evidence for this hypothesis was very weak. There was no group by emotion interaction in the univariate analysis, and the CD-TD classifier performed less well than the HCU-TD classifier only, suggesting that differences between $\mathrm{CD} / \mathrm{HCU}$ and $\mathrm{CD} / \mathrm{LCU}$ were mostly quantitative rather than qualitative. However, the most relevant features in the HCU-TD and HCU-LCU models were fear and sadness, respectively; whereas surprise was most relevant for the LCU-TD model. Difficulties with fearful and sad facial expressions were, thus, the strongest markers of $\mathrm{CD} / \mathrm{HCU}$, but not $\mathrm{CD} / \mathrm{LCU}$, in individual youths.

\section{Prevalence of emotion recognition difficulties in CD and implications for its theoretical understanding}

These findings are in line with previous meta-analytic work [19], and suggest that facial emotion recognition difficulties are common in $\mathrm{CD} / \mathrm{HCU}$ and are generally not restricted to fear and sadness. In contrast to previous findings from an all-female sample [10], but in line with work by Kohls et al. [6] and Fairchild et al. [14], difficulties were seen in female as well as male youths. However, emotion recognition difficulties in $\mathrm{CD}$ were by no means universal, especially for the CD/LCU group, who did not differ significantly from the TD group in univariate analyses. Indeed, the effect size for the main effect of group in the univariate analyses was small, and in multivariate analyses, approximately $50 \%$ of youths with $\mathrm{CD}$ were misclassified. By contrast, TD youths were misclassified far less frequently. This pattern of misclassification implies an 'imbalanced' overlap between groups, with youths in the $\mathrm{CD}$ groups more likely to resemble TD youths than vice versa. These findings suggest that while poor emotion recognition ability is considerably more common in CD than in TD youths, many youths with CD exhibit 'normal' emotion recognition abilities, in line with recent research in this area $[6,7,10]$.

Group-level differences between $\mathrm{CD} / \mathrm{HCU}$ and $\mathrm{CD} / \mathrm{LCU}$ did not emerge in the present study, in line with a minority of previous studies [7,11]. These divergent findings might be partially explained by differences in study design, criteria for $\mathrm{CD} / \mathrm{HCU}$, and choice of control group in previous research (CD/LCU or TD youths). However, studies that do report poorer emotion recognition in $\mathrm{CD} / \mathrm{HCU}$ often report particular difficulties with negative emotions, especially fear and sadness [12-14]. This is notable because in our AngleGMLVQ analyses, fear and sadness recognition difficulties were the most relevant markers of $\mathrm{CD} / \mathrm{HCU}$, despite the lack of significant group differences. Feature relevance scores were based on classifiers that achieved at least $60 \%$ classification accuracy, which implies that when the classifiers were able to discriminate between $\mathrm{CD} / \mathrm{HCU}$ and the other groups, they did so principally on the basis of fear and sadness recognition. This finding may help to clarify the previous literature, where group differences are not always present, but when they are, they are strongest for fear and sadness.

Importantly, these results also highlight a discrepancy between theoretical descriptions of $\mathrm{CD} / \mathrm{HCU}$, namely that these are youths who have an impaired ability to recognise and respond appropriately to distress in others [5], and common clinical or research definitions of $\mathrm{CD} / \mathrm{HCU}$, which are based directly on high scores on CU trait measures. The 
latter are agnostic with regard to aetiology and underlying neurocognitive characteristics. The lack of precise clinical criteria and measures for $\mathrm{CD} / \mathrm{HCU}$ is obviously a major hindrance to the literature here, since it is possible that the discrepancy between clinical presentation $(\mathrm{CD} / \mathrm{HCU})$ and the assumed underlying psychopathology (emotion recognition deficits) simply reflects an inappropriate threshold for $\mathrm{CD} / \mathrm{HCU}$. This might be the case if $\mathrm{CU}$ traits and emotion recognition have a non-linear relationship, with emotion recognition deficits manifesting only at the extreme upper end of the CU trait spectrum (see, e.g. [35]). Alternatively, if the relationship is linear, the precise cut-off is less crucial. However, we note that an approach based more closely on the Diagnostic and Statistical Manual of Mental Disorders Limited Prosocial Emotions criteria, and using a sample that overlaps with the present one, also failed to identify any association between CU traits and emotion recognition performance [7]. These considerations around clinical threshold notwithstanding, the results of the current study raise a broader question as to whether CU traits constitute the psychopathology itself (thus, warranting a behavioural diagnosis), or are an indirect measure of an underlying psychopathology such as emotion recognition deficits. Our findings do not support any particular position on this point, but they do highlight the possibility that these two approaches will not identify the same subset of youths. Similar discrepancies were previously observed by Kohls et al. $[6,7]$ in the context of CD more generally, and they merit serious consideration both for future research and clinical approaches to $\mathrm{CD}$.

\section{Emotional intensity and recognition accuracy}

Finally, an interesting and unexpected finding in the present study was the pattern of feature relevance for the different emotion intensities in the classifier models. Somewhat counterintuitively, group differences in recognition accuracy were larger for the high-intensity emotions compared to the low-intensity emotions, and the feature relevance analyses confirmed that difficulties with high-intensity emotions were more relevant markers of CD subtypes than were low-intensity emotions. The reason for this is not clear, and further research with a wider range of emotional intensities [18, 36], as well as neutral faces, will be needed to confirm this finding. However, it suggests that TD youths benefit more from increased emotional intensity than do youths with $\mathrm{CD}$, perhaps indicating that the difficulties seen in CD are not 'threshold' effects but rather more absolute difficulties with recognising emotions.

\section{Strengths and limitations}

This study has several strengths, including a large, well-characterised, mixed-sex sample and the use of multivariate analyses. However, some limitations should be noted. First, the Emotion Hexagon task uses static expressions, and the identity of the face is the same across all expressions. The ecological validity of the task would be improved by the inclusion of a range of facial identities, as well as dynamic stimuli (although recent work indicates similar effects for dynamic stimuli [21]). Second, the Emotion Hexagon task does not include neutral expressions, which complicates the interpretation of errors. Third, the $\mathrm{CD} / \mathrm{HCU}$ group in the current sample also had more severe $\mathrm{CD}$ than the $\mathrm{CD} / \mathrm{LCU}$ group. Although this is clinically typical of $\mathrm{CD} / \mathrm{HCU}$, it is an important consideration, because some neuroimaging studies of amygdala reactivity to emotional faces report a stronger association with $\mathrm{CD}$ severity than with CU traits [37]. Fourth, TD participants had no current psychiatric disorders, and were, thus, not fully representative of the general population. This is notable due to the complex associations between internalising disorders and emotion recognition difficulties [36, 38]. Although we repeated our analyses after controlling for comorbidities and found no substantial changes, the nature of our TD group might still have led to an over- or underestimate of 'normative' emotion recognition abilities. Fifth, the sample spanned a large age range (9-18 years), and we were not able to investigate any potential developmental differences across this age range. Finally, we would have ideally validated our classifiers by further testing them on a completely independent dataset, but this was not possible due to a lack of available datasets.

\section{Summary and conclusions}

In summary, these findings indicate that, compared to TD youths, youths with $\mathrm{CD} / \mathrm{HCU}$ frequently have difficulty recognising facial emotional expressions. However, differences between $\mathrm{CD} / \mathrm{HCU}$ and $\mathrm{CD} / \mathrm{LCU}$ were smaller and not significant. Indeed, there was substantial overlap between all three groups, emphasising again the heterogeneous nature of $\mathrm{CD}$ as a diagnostic category. Nonetheless, the most relevant emotions for distinguishing $\mathrm{CD} / \mathrm{HCU}$ from TD and from $\mathrm{CD} / \mathrm{LCU}$ in classification analyses were fear and sadness, indicating that when youths with $\mathrm{CD} / \mathrm{HCU}$ do differ from other youths, they tend to differ most on recognition of fear and sadness. These findings highlight the existence of heterogeneous abilities within both $\mathrm{CD} / \mathrm{HCU}$ and $\mathrm{CD} / \mathrm{LCU}$, and the need to establish what (if any) level of difficulty is reliably associated with $\mathrm{CD}$, and $\mathrm{CD} / \mathrm{HCU}$ in particular. Longitudinal research designs will also be necessary to elucidate whether, or how, emotion recognition difficulties contribute to the development of antisocial behaviour and aggression in adolescence. 
Supplementary Information The online version contains supplementary material available at https://doi.org/10.1007/s00787-021-01893-5.

Acknowledgements We thank all members of the FemNAT-CD consortium for their contributions to the project. In particular, we thank Marietta Kirchner for her work on the imputation of missing data. Finally, we would like to thank our participants, their families, and the many professionals who gave their time generously.

Author contributions RP collected data, conducted all analyses, and wrote the manuscript, under the supervision of SADB, PR, and PT. GK pre-processed data from the Emotion Hexagon task. PT developed the machine learning classifier and advised on its use. All other authors contributed substantially to data collection, study design, and/ or preparation of the manuscript. All authors read and approved the final manuscript.

Funding This study was conducted by the FemNAT-CD consortium (Neurobiology and Treatment of Adolescent Female Conduct Disorder: The Central Role of Emotion Processing, coordinator Christine M. Freitag). This collaborative project is funded by the European Commission under the 7th Framework Health Program, Grant Agreement no. 602407. Ruth Pauli was supported by the Biotechnology and Biological Sciences Research Council's Midlands Integrative Biosciences Training Partnership (BBSRC MIBTP). During the writing of the manuscript, Stephane A. De Brito was supported by a short-term Invitational Fellowship from the Japanese Society for the Promotion of Science (JSPS - S19103) and an International Academic Fellowship from the Leverhulme Trust (IAF-2019-032).

Data availability Data are available from the authors on request.

Code availability Code is available from the authors on request.

\section{Declarations}

Conflict of interest Christine M. Freitag receives royalties for books on attention-deficit/hyperactivity disorder and autism spectrum disorder. She has served as consultant to Desitin and Roche. No other authors report any conflicts of interest.

Ethics approval The FemNAT-CD project received ethical approval from the relevant local ethics committees, as follows: Aachen: Ethik Kommission Medizinische Fakultät der Rheinisch Westfälischen Technischen Hochschule Aachen (EK027/14). Amsterdam: Medisch Etische Toetsingscommissie (2014.188). Athens: Election Committee of the First Department of Psychiatry, Eginition University Hospital (641/9.11.2015). Barcelona: Child and Adolescent Mental Health-University Hospital Mutua Terrassa (acta 12/13). Basel: Ethik Kommission Nordwest- und Zentralschweiz (EKNZ 336/13). Bilbao: Hospital del Basurto. Birmingham and Southampton: University Ethics Committee and National Health Service Research Ethics Committee (NRES Committee West Midlands, Edgbaston; REC reference 3/WM/0483). Dublin: SJH/AMNCH Research Ethics Committee (2014/04/Chairman (3)). Frankfurt: Ethik Kommission Medizinische Fakultät Goethe Universität Frankfurt am Main (445/13). Szeged (Hungary): Egészségügyi Tudományos Tanács Humán Reprodukciós Bizottság (CSR/039/00392-3/2014). This study was conducted in accordance with the ethical standards of the 1964 Declaration of Helsinki and its later amendments.

Consent to participate All participants over the legal age of consent (16 or 18 years) in their country provided written informed consent to participate. Participants under the age of consent provided written informed assent, with written informed consent provided by a parent or guardian.

Consent for publication All authors consented to the publication of this paper.

Open Access This article is licensed under a Creative Commons Attribution 4.0 International License, which permits use, sharing, adaptation, distribution and reproduction in any medium or format, as long as you give appropriate credit to the original author(s) and the source, provide a link to the Creative Commons licence, and indicate if changes were made. The images or other third party material in this article are included in the article's Creative Commons licence, unless indicated otherwise in a credit line to the material. If material is not included in the article's Creative Commons licence and your intended use is not permitted by statutory regulation or exceeds the permitted use, you will need to obtain permission directly from the copyright holder. To view a copy of this licence, visit http://creativecommons.org/licenses/by/4.0/.

\section{References}

1. Cleckley H (1976) The mask of sanity, 5th edn. Mosby

2. American Psychiatric Association (2013) Diagnostic and statistical manual of mental disorders (DSM-5). American Psychiatric Association

3. Fanti KA, Frick PJ, Georgiou S (2008) Linking callous-unemotional traits to instrumental and non-instrumental forms of aggression. J Psychopathol Behav Assess 31(4):285. https://doi.org/10. 1007/s10862-008-9111-3

4. Frick PJ, Stickle TR, Dandreaux DM, Farrell JM, Kimonis ER (2005) Callous-unemotional traits in predicting the severity and stability of conduct problems and delinquency. J Abnorm Child Psychol 33(4):471-487. https://doi.org/10.1007/ s10648-005-5728-9

5. Blair J (2013) The neurobiology of psychopathic traits in youths. Nat Rev Neurosci 14(11):786-799. https://doi.org/10.1038/nrn35 77

6. Kohls G et al (2020) Investigating sex differences in emotion recognition, learning, and regulation among youths with conduct disorder. J Am Acad Child Adolesc Psychiatry 59(2):263-273. https://doi.org/10.1016/j.jaac.2019.04.003

7. Kohls G et al (2020) Neuropsychological subgroups of emotion processing in youths with conduct disorder. Front Psychiatry. https://doi.org/10.3389/fpsyt.2020.585052

8. Blair J, Colledge E, Murray L, Mitchell DGV (2001) A selective impairment in the processing of sad and fearful expressions in children with psychopathic tendencies. J Abnorm Child Psychol 29(6):491-498. https://doi.org/10.1023/A:1012225108281

9. Sully K, Sonuga-Barke EJS, Fairchild G (2015) The familial basis of facial emotion recognition deficits in adolescents with conduct disorder and their unaffected relatives. Psychol Med 45(9):19651975. https://doi.org/10.1017/S0033291714003080

10. Pajer K, Leininger L, Gardner W (2010) Recognition of facial affect in girls with conduct disorder. Psychiatry Res 175(3):244 251. https://doi.org/10.1016/j.psychres.2009.06.003

11. Rehder PD, Mills-Koonce WR, Willoughby MT, Garrett-Peters P, Wagner NJ (2017) Emotion recognition deficits among children with conduct problems and callous-unemotional behaviors. Early Child Res Q 41:174-183. https://doi.org/10.1016/j.ecresq.2017. 07.007

12. Stevens D, Charman T, Blair RJR (2001) Recognition of emotion in facial expressions and vocal tones in children with psychopathic 
tendencies. J Genet Psychol 162(2):201-211. https://doi.org/10. 1080/00221320109597961

13. Fairchild G, Goozen SHMV, Calder AJ, Stollery SJ, Goodyer IM (2009) Deficits in facial expression recognition in male adolescents with early-onset or adolescence-onset conduct disorder. J Child Psychol Psychiatry 50(5):627-636. https://doi.org/10. 1111/j.1469-7610.2008.02020.x

14. Fairchild G, Stobbe Y, van Goozen SHM, Calder AJ, Goodyer IM (2010) Facial expression recognition, fear conditioning, and startle modulation in female subjects with conduct disorder. Biol Psychiatry 68(3):272-279. https://doi.org/10.1016/j.biopsych. 2010.02.019

15. Hartmann D, Schwenck C (2020) Emotion processing in children with conduct problems and callous-unemotional traits: an investigation of speed, accuracy, and attention. Child Psychiatry Hum Dev. https://doi.org/10.1007/s10578-020-00976-9

16. Hoppenbrouwers SS, Munneke J, Kooiman KA, Little B, Neumann CS, Theeuwes J (2017) Fearful faces do not lead to faster attentional deployment in individuals with elevated psychopathic traits. J Psychopathol Behav Assess 39(4):596-604. https://doi. org/10.1007/s10862-017-9614-x

17. Blair RJR, Coles M (2000) Expression recognition and behavioural problems in early adolescence. Cogn Dev 15(4):421-434. https://doi.org/10.1016/S0885-2014(01)00039-9

18. Bowen KL, Morgan JE, Moore SC, van Goozen SHM (2014) Young Offenders' emotion recognition dysfunction across emotion intensities: explaining variation using psychopathic traits, conduct disorder and offense severity. J Psychopathol Behav Assess 36(1):60-73. https://doi.org/10.1007/s10862-013-9368-z

19. Dawel A, O'Kearney R, McKone E, Palermo R (2012) Not just fear and sadness: meta-analytic evidence of pervasive emotion recognition deficits for facial and vocal expressions in psychopathy. Neurosci Biobehav Rev 36(10):2288-2304. https://doi.org/ 10.1016/j.neubiorev.2012.08.006

20. Schwenck $C$ et al (2012) Empathy in children with autism and conduct disorder: group-specific profiles and developmental aspects. J Child Psychol Psychiatry 53(6):651-659. https://doi. org/10.1111/j.1469-7610.2011.02499.x

21. Martin-Key NA, Graf EW, Adams WJ, Fairchild G (2018) Facial emotion recognition and eye movement behaviour in conduct disorder. J Child Psychol Psychiatry 59(3):247-257. https://doi.org/ 10.1111/jcpp. 12795

22. Bunte K, Baranowski ES, Arlt W, Tino P (2016) Relevance learning vector quantization in variable dimensional spaces. New Challenges in Neural Computation (NC 2), pp. 20-23.

23. Pauli R, et al. (2020) Positive and negative parenting in conduct disorder with high versus low levels of callous-unemotional traits. Dev Psychopathol. [Online]. https://researchportal.bath.ac.uk/en/ publications/positive-and-negative-parenting-in-conduct-disorderwith-high-ver. (Accessed 27 Jul 2020.)

24. Freitag CM et al (2018) Conduct disorder in adolescent females: current state of research and study design of the FemNAT-CD consortium. Eur Child Adolesc Psychiatry 27(9):1077-1093. https://doi.org/10.1007/s00787-018-1172-6
25. Essau CA, Sasagawa S, Frick PJ (2006) Callous-unemotional traits in a community sample of adolescents. Assessment 13(4):454469. https://doi.org/10.1177/1073191106287354

26. Kaufman J et al (1997) Schedule for affective disorders and schizophrenia for school-age children-present and lifetime version (K-SADS-PL): initial reliability and validity data. J Am Acad Child Adolesc Psychiatry 36(7):980-988. https://doi.org/10.1097/ 00004583-199707000-00021

27. Wechsler D (1999) Wechsler abbreviated scale of intelligence. Psychological Corporation

28. Wechsler D (2008) Wechsler adult intelligence scale-fourth edition (WAIS-IV). Psychological Corporation

29. Petersen AC, Crockett L, Richards M, Boxer A (1988) A selfreport measure of pubertal status: reliability, validity, and initial norms. J Youth Adolesc 17(2):117-133. https://doi.org/10.1007/ BF01537962

30. Calder AJ (1996) Facial emotion recognition after bilateral amygdala damage: differentially severe impairment of fear. Cogn Neuropsychol 13(5):699-745. https://doi.org/10.1080/0264329963 81890

31. Fontaine JRJ, Scherer KR, Roesch EB, Ellsworth PC (2007) The world of emotions is not two-dimensional. Psychol Sci 18(12):1050-1057. https://doi.org/10.1111/j.1467-9280.2007. 02024.x

32. Baccianella S, Esuli A, Sebastiani F (2009) Evaluation measures for ordinal regression," in 2009 ninth international conference on intelligent systems design and applications. 283-287. https://doi. org/10.1109/ISDA.2009.230.

33. Fouad S, Tino P (2012) Adaptive metric learning vector quantization for ordinal classification. Neural Comput 24(11):2825-2851. https://doi.org/10.1162/NECO_a_00358

34. Schneider P, Biehl M, Hammer B (2009) Adaptive relevance matrices in learning vector quantization. Neural Comput 21(12):3532-3561

35. Abu-Akel A, Heinke D, Gillespie SM, Mitchell IJ, Bo S (2015) Metacognitive impairments in schizophrenia are arrested at extreme levels of psychopathy: the cut-off effect. J Abnorm Psychol 124(4):1102-1109. https://doi.org/10.1037/abn0000096

36. Short RML, Sonuga-Barke EJS, Adams WJ, Fairchild G (2016) Does comorbid anxiety counteract emotion recognition deficits in conduct disorder? J Child Psychol Psychiatry 57(8):917-926. https://doi.org/10.1111/jcpp.12544

37. Dotterer HL et al (2020) Clarifying the link between amygdala functioning during emotion processing and antisocial behaviors versus callous-unemotional traits within a population-based community sample. Clin Psychol Sci 8(5):918-935. https://doi.org/10. $1177 / 2167702620922829$

38. Schepman K, Taylor E, Collishaw S, Fombonne E (2012) Face emotion processing in depressed children and adolescents with and without comorbid conduct disorder. J Abnorm Child Psychol 40(4):583-593. https://doi.org/10.1007/s10802-011-9587-2

\section{Authors and Affiliations}

Ruth Pauli ${ }^{1}$. Gregor Kohls ${ }^{2,3} \cdot$ Peter Tino $^{4} \cdot$ Jack C. Rogers $^{1,5} \cdot$ Sarah Baumann $^{6} \cdot$ Katharina Ackermann $^{7,8}$. Anka Bernhard ${ }^{7} \cdot$ Anne Martinelli $^{7} \cdot$ Lucres Jansen $^{9} \cdot$ Helena Oldenhof $^{9} \cdot$ Karen Gonzalez-Madruga $^{10}$. Areti Smaragdi ${ }^{11} \cdot$ Miguel Angel Gonzalez-Torres ${ }^{12} \cdot$ Iñaki Kerexeta-Lizeaga ${ }^{12} \cdot$ Cyril Boonmann $^{13} \cdot$ Linda Kersten $^{13}$. Aitana Bigorra ${ }^{14}$. Amaia Hervas ${ }^{14}$. Christina Stadler ${ }^{13}$. Aranzazu Fernandez-Rivas ${ }^{12}$. Arne Popma ${ }^{9}$. Kerstin Konrad ${ }^{2,15} \cdot$ Beate Herpertz-Dahlmann $^{6} \cdot$ Graeme Fairchild $^{16} \cdot$ Christine M. Freitag $^{7} \cdot$ Pia Rotshtein $^{1}$. Stephane A. De Brito ${ }^{1}$ 
1 Centre for Human Brain Health, School of Psychology, University of Birmingham, Birmingham B15 2TT, UK

2 Child Neuropsychology Section, Department of Child and Adolescent Psychiatry, Psychosomatics and Psychotherapy, RWTH Aachen University, Aachen, Germany

3 Department of Child and Adolescent Psychiatry, Faculty of Medicine, TU, Dresden, Germany

4 School of Computer Science, University of Birmingham, Birmingham, UK

5 Institute for Mental Health, School of Psychology, University of Birmingham, Birmingham, UK

6 Department of Child and Adolescent Psychiatry, Psychosomatics and Psychotherapy, University Hospital RWTH Aachen, Aachen, Germany

7 Department of Child and Adolescent Psychiatry, Psychosomatics and Psychotherapy, Goethe University, University Hospital Frankfurt, Frankfurt am Main, Germany
8 Faculty of Education, Hamburg University, Hamburg, Germany

9 Department of Child and Adolescent Psychiatry, VU University Medical Center, Amsterdam, the Netherlands

10 Department of Child and Adolescent Psychiatry, Institute of Psychiatry, Psychology and Neuroscience, Kings College London, London, UK

11 Child Development Institute, Toronto, Canada

12 Psychiatric Service, Basurto University Hospital, Bilbao, Spain

13 Department of Child and Adolescent Psychiatry, University Psychiatric Hospitals, University of Basel, Basel, Switzerland

14 University Hospital Mutua Terrassa, Barcelona, Spain

15 JARA-Brain Institute II, Molecular Neuroscience and Neuroimaging, RWTH Aachen and Research Centre Juelich, Juelich, Germany

16 Department of Psychology, University of Bath, Bath, UK 